\title{
Permanencia laboral de otorrinolaringólogos en el servicio público luego de egresar de la especialidad y factores asociados a ésta
}

\author{
Permanence of otolaryngologists in public service after graduating from the \\ specialty and factors associated with this
}

Felipe Cardemil M1,2, Tamara Barría $\mathrm{E}^{3}$, Maritza Rahal E'.

\begin{abstract}
RESUMEN
Introducción: En Chile existe un problema en la distribución de médicos otorrinolaringólogos (ORL) entre el sistema público (SP) y privado. Además, recién a partir del presente año existe un sistema formal de vínculo entre algunos especialistas recién egresados y el $S P$, por lo que hay un déficit de ORL en éste.

Objetivo: Describir el grupo de ORL egresados en la última década y evaluar permanencia en SP de salud.

Material y método: Estudio de corte transversal. Se analizaron los titulados como ORL en el período 2003-2012. Se evaluó mediante un cuestionario online si trabajaba en el SP, carga horaria, tiempo de permanencia, razones de ingreso y retiro del SP, y ventajas y desventajas de éste.

Resultados: Hubo 114 egresados. La cantidad de especialistas formados se ha mantenido en alrededor de 12 por año entre todos los centros del país. Del total, 43 contestaron el cuestionario, de los cuales actualmente trabajan en hospitales del SP el 58,1\%. El tipo de contrato en el SP disminuyó con el tiempo en $26,2 \%$ de los casos. El promedio de tiempo trabajado en el SP fue 3 años 10 meses. Dentro de las principales ventajas de trabajar en el SP se mencionan la experiencia quirúrgica, complejidad de patologías y trabajo en equipo, y dentro de las desventajas se menciona la baja remuneración, y poca disponibilidad infraestructura, instrumental y pabellones.

Discusión: Es importante considerar estos factores al diseñar las políticas de vínculo y retención de ORL en el sector público de salud.
\end{abstract}

Palabras clave: Otorrinolaringólogos, sector público de salud, permanencia.

\begin{abstract}
Introduction: In Chile there is a problem in the distribution of ENT surgeons between the public (PHS) and private health system. Furthermore, only since this year there is a formal link between some recent ENT surgeons graduates, so there is a deficit of ENT in PHS.

Aim: To describe the group of ENT graduates in the last decade and evaluate their permanence working in the PHS.
\end{abstract}

\footnotetext{
${ }^{1}$ Servicio de Otorrinolaringología, Hospital Barros Luco Trudeau - Facultad de Medicina, Universidad de Chile.

2 Programa de Doctorado en Salud Pública, Escuela de Salud Pública, Universidad de Chile.

${ }^{3}$ Médico Cirujano, Escuela de Salud Pública, Universidad de Chile.
} 
Material and method: Cross sectional study. There were analyzed the ORL graduates in the period 2003-2012. We evaluated by an email questionnaire if working at PHS, workload, time worked, reasons why linking with PHS, and advantages and disadvantages of this.

Results: There were 114 graduates. The number of trained specialists has remained at about 12 per year from all teaching centers of the country. Of the total, 43 completed the questionnaire, of whom currently work in PHS hospitals 58.1\%. The type of contract in the PHS decreased with time in $26.2 \%$ of cases. The average time worked in the PHS was 3 years 10 months. Among the main advantages of working in the PHS, they mentioned gain surgical experience, complexity of pathologies and teamwork, and within the disadvantages mentioned low payment, and poor availability of infrastructure, instrumental and operating rooms.

Discussion: It is important to consider these factors when designing policies of linking and retention of ENT surgeons in public health system hospitals.

Key words: ENT surgeons, public health system hospitals, permanence.

\section{INTRODUCCIÓN}

En nuestro país la distribución de médicos otorrinolaringólogos ( $\mathrm{ORL}$ ) en las diferentes regiones es manifiestamente desigual, existiendo una concentración de ellos en las regiones Metropolitana, $\mathrm{V}$ y VIII, y escasez de ellos en general en las otras. Esto, a su vez, está asociado a una gran disparidad en la cantidad de ORL entre el sistema público (SP) y privado ${ }^{1}$.

La necesidad de incrementar el número de médicos especialistas se ha basado en la necesidad de cerrar las brechas de atención especializada en el país, determinadas por los estudios de la Secretaría Técnica del Plan de Acceso Universal de Garantías Explícitas (AUGE, 2004) y del Banco Mundial (2009). La falta de especialistas señalada en estos estudios contrasta con la existencia en el país de un franco predominio de médicos especialistas sobre los generales, alcanzando los especialistas el $56 \%$ del total en el país en el año 2004, y al $64 \%$ del total del sistema público y al $81 \%$ en los servicios de salud ${ }^{2-4}$. Esto se asocia a que la tendencia preponderante en la actualidad, tanto de médicos como estudiantes de medicina, es preferir el sistema de salud privado para desempeñarse. En consecuencia, la política pública de recursos humanos debe orientarse a la captación y retención de especialistas ${ }^{5}$. Por este motivo, en nuestro país se estimó que entre el año 2007 y 2010 ingresaron 1.600 médicos a programas de formación de especialistas con obligación de retorno en el servicio público ${ }^{5}$.
Se reporta que en nuestro país otorrinolaringología es una de las especialidades con menor cantidad de médicos con retorno al servicio público luego de terminar la especialización ${ }^{5}$. De las diferentes vías de acceso a los programas de especialización, sólo la mitad de los casos tienen la obligación de trabajar en el sistema de salud público. En consecuencia, a los 7 años luego de su ingreso a dicho sistema, sólo el $60 \%$ de ellos permanece en él 6 . Por esta razón, el ingreso y la mantención al sector público de los ORL no está asegurado. Existen numerosos factores que condicionan las opciones laborales de los profesionales médicos hacia los sectores público o privado. Los principales focos de interés atribuidos al sector público son la inserción en un equipo profesional que atiendan una gran variedad de problemas de salud, el tipo de práctica laboral, las posibilidades de desarrollo profesional que se desprende de ella, y estabilidad de ingresos ${ }^{7}$.

Es por esto, que nuestro objetivo fue describir el grupo de ORL egresados en la última década, evaluar permanencia en sector público de salud, y los motivos que han influido en ésta.

\section{MATERIAL Y MÉTODO}

Estudio de corte transversal. Se analizaron los titulados como ORL en programas de postítulo acreditados de universidades chilenas en el período 2003-2012, en base a los datos disponibles en la Sociedad Chilena de Otorrinolaringología, Medici- 
na y Cirugía de Cabeza y Cuello (SOCHIORL). Se evaluó mediante un cuestionario diseñado ad hoc a través de un servicio de encuestas online si trabajaba actualmente o había trabajado en el pasado en hospitales públicos dependientes del Ministerio de Salud (MINSAL), la carga horaria, el tiempo de permanencia en caso que hubiera renunciado, y las razones de ingreso y retiro del sector público. Las encuestas fueron anónimas.

Los datos se analizaron con Stata 11. Se usaron descriptores estadísticos generales, como promedios y frecuencias absolutas y relativas. Para evaluar asociación se utilizó test exacto de Fisher. Se consideró significativo un valor de $p$ igual 0 menor a 0,05 .

\section{RESULTADOS}

En base a los datos de la SOCHIORL, se observaron 114 egresados, correspondientes a 26 $(22,8 \%)$ mujeres y $88(77,2 \%)$ hombres. La cantidad de especialistas formados se ha mantenido relativamente constante durante este período, con 12 especialistas por año en el 2003 y 12 en el 2012, con pequeñas variaciones interanuales (Figura 1). Del total de 114 ORL, actualmente trabajan en algún hospital público dependiente del MINSAL el $57 \%$ de ellos.

Se logró obtener el contacto de 97 egresados, de los cuales contestaron el cuestionario online 43
$\mathrm{ORL}$, lo que corresponde a la muestra del presente estudio. De los encuestados, 13 (30,2\%) fueron mujeres. El promedio de edad actual de los encuestados fue de 35 años, y el promedio de edad al titularse fue 29 años. En cuanto a la vía de ingreso, el $54,8 \%$ fueron recién egresados, el $19,1 \%$ fueron de generaciones anteriores, y $26,2 \%$ ingresaron vía retorno de la etapa de destinación y formación (EDF o "generales de zona"). Respecto al financiamiento, el 38,5\% fue autofinanciado, 33,3\% financiado por el MINSAL (EDF o becado primario con retorno a un hospital posterior a la finalización del programa), y $28,2 \%$ fue financiado por otra institución (Universidad, Fuerzas Armadas, u otra).

De la muestra de 43 encuestados, actualmente trabajan alguna parte de su jornada laboral en hospitales del MINSAL el 58,1\%. El promedio de tiempo trabajado en el SP fue 3 años 10 meses. El 20,5\% de los cargos contratados fueron de 11 $\mathrm{hrs} /$ semanales y el $51,3 \%$ de $22 \mathrm{hrs} /$ semanales, y el $20,5 \%$ de $33 \mathrm{hrs} / \mathrm{semanales.} \mathrm{El} \mathrm{tipo} \mathrm{de} \mathrm{contrato}$ en el SP disminuyó con el tiempo en $26,2 \%$ de los casos, y aumentó su carga horaria sólo en 2,4\%. No presentaron variaciones en su carga horaria el $64,3 \%$ de los encuestados. El tiempo promedio en el que se produjo la disminución de la carga horaria fue de 3 años y 4 meses. Existió una asociación significativa entre $\mathrm{ORL}$ que variaron su carga horaria $(28,3 \%)$ y carga horaria actual $(p=0,02)$.

Al preguntar por los motivos de ingreso a trabajar a hospitales públicos, se menciona "Ad-

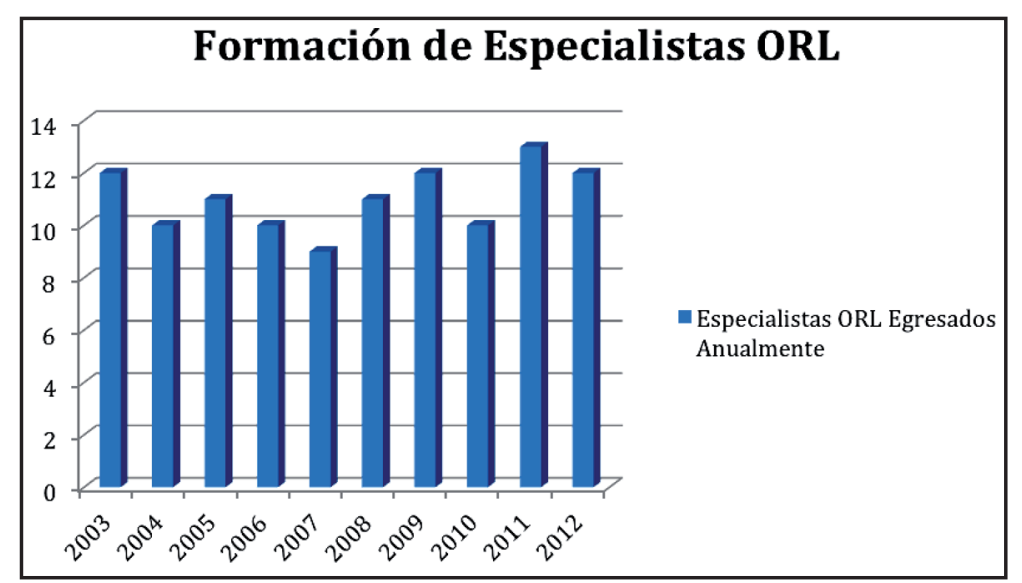

Figura 1. Cantidad de otorrinolaringólogos egresados de programas de postítulo en Chile durante el período 2003-2012. 
quirir experiencia/Formación continua" (29,4\%), "Trabajo en el hospital como labor social" (27,5\%), y "Dinámica de trabajo en equipo" $(11,8 \%)$ como las 3 más frecuentes (Tabla 1). Dentro de los motivos de renuncia al SP se registró "Falta de recursos humanos, físicos o técnicos" $(29,4 \%)$, "Poca disponibilidad de pabellón" (17,6\%), y "Baja remuneración" $(17,6 \%)$ como los más frecuentes (Tabla 2).

Tabla 1. Motivo de ingreso a trabajar a sector público de salud en otorrinolaringólogos ( $n=43$ )

\begin{tabular}{|l|c|}
\hline Motivo & $\begin{array}{c}\text { Frecuencia } \\
(\%)\end{array}$ \\
\hline $\begin{array}{l}\text { Adquirir experiencia/Formación } \\
\text { continua }\end{array}$ & 29,4 \\
\hline $\begin{array}{l}\text { Trabajo en el hospital como } \\
\text { labor social }\end{array}$ & 27,5 \\
\hline Dinámica de trabajo en equipo & 11,8 \\
\hline Docencia & 9,8 \\
\hline $\begin{array}{l}\text { Variedad y complejidad de } \\
\text { patologías en hospital }\end{array}$ & 5,9 \\
\hline $\begin{array}{l}\text { Obliga ción de ret orno } \\
\text { posespecialización }\end{array}$ & 3,9 \\
\hline Oportunidad quirúrgica & 3,9 \\
\hline Otros & 7,8 \\
\hline
\end{tabular}

Tabla 2. Motivo de renuncia a sector público de salud en otorrinolaringólogos $(n=43)$

\begin{tabular}{|l|c|}
\hline Motivo & $\begin{array}{c}\text { Frecuencia } \\
(\%)\end{array}$ \\
\hline $\begin{array}{l}\text { Falta de recursos humanos, físicos } \\
\text { o técnicos }\end{array}$ & 29,4 \\
\hline Poca disponibilidad de pabellón & 17,6 \\
\hline Baja remuneración & 17,6 \\
\hline Poca flexibilidad horaria & 11,8 \\
\hline Alta presión asistencial & 11,8 \\
\hline Mala organización del sistema & 5,9 \\
\hline Poco apoyo institucional & 5,9 \\
\hline Desarrollar subespecialidad & 5,9 \\
\hline
\end{tabular}

Dentro de las ventajas de trabajar en el sector público mencionadas más frecuentemente están "Adquirir experiencia" (18,8\%), "Variedad y com- plejidad de patologías" (15,6\%), y "Dinámica de trabajo en equipo" (14\%). Al preguntar las desventajas se menciona con más frecuencia "Baja remuneración" (32,6\%), "Falta de instrumental e infraestructura" (22,1\%), y "Poca disponibilidad y suspensión de pabellones” (12,8\%) (Tabla 3).

Tabla 3. Ventajas y desventajas de trabajar en sector público de salud mencionadas por otorrinolaringólogos ( $\mathrm{n}=43$ )

\begin{tabular}{|c|c|c|}
\hline \multirow[t]{9}{*}{ Ventajas } & & Frecuencia (\%) \\
\hline & Adquirir experiencia & 18,8 \\
\hline & $\begin{array}{l}\text { Variedad y } \\
\text { complejidad de } \\
\text { patologías }\end{array}$ & 15,6 \\
\hline & $\begin{array}{l}\text { Dinámica de trabajo } \\
\text { en equipo }\end{array}$ & 14 \\
\hline & $\begin{array}{l}\text { Trabajo en el } \\
\text { hospital como labor } \\
\text { social }\end{array}$ & 13,5 \\
\hline & Docencia & 11,5 \\
\hline & $\begin{array}{l}\text { Contrato y } \\
\text { estabilidad laboral }\end{array}$ & 8,3 \\
\hline & $\begin{array}{l}\text { Oportunidad } \\
\text { quirúrgica }\end{array}$ & 4,2 \\
\hline & Otros & 13,5 \\
\hline \multirow[t]{9}{*}{ Desventajas } & & Frecuencia (\%) \\
\hline & Baja remuneración & 32,6 \\
\hline & $\begin{array}{l}\text { Falta de } \\
\text { instrumental e } \\
\text { infraestructura }\end{array}$ & 22,1 \\
\hline & $\begin{array}{l}\text { Poca disponibilidad } \\
\text { y suspensión de } \\
\text { pabellones }\end{array}$ & 12,8 \\
\hline & $\begin{array}{l}\text { Alta carga } \\
\text { asistencial }\end{array}$ & 9,3 \\
\hline & Riesgo médico legal & 3,5 \\
\hline & $\begin{array}{l}\text { Falta de estímulos } \\
\text { laborales y } \\
\text { profesionales }\end{array}$ & 3,5 \\
\hline & Burocracia & 3,5 \\
\hline & Otros & 13,5 \\
\hline
\end{tabular}

Al analizar la cantidad de médicos que permanecen trabajando en el sector público según el año de egreso de la especialidad, se aprecia que hay una relación inversa respecto a la antigüedad de 
egreso. Esto se aprecia en la Tabla 4, en la que se observa que de los ORL egresados el 2003, sólo el $27,3 \%$ permanece trabajando en el sector público, proporción que alcanza al $62,5 \%$ para los egresados del año 2007, y al 100\% para los egresados en la última promoción evaluada (2012), siguiendo una tendencia a mayor proporción de trabajo en el sector público para los egresados de años más recientes. Esta asociación fue significativa ( $p=0,003$ ).

\section{DISCUSIÓN}

Existe un déficit relativo de especialistas en el sistema de salud público, por lo que se han propuesto diversos objetivos para corregir esto sin apelar a un aumento desregulado de médicos formados. Se ha considerado mejorar el financiamiento del sistema de formación tanto para las Universidades como para los centros asistenciales donde se realiza en forma tutorial esta formación, aumentar progresivamente la vía de formación como especialista rápidamente después del egreso de las Escuelas de Medicina (idealmente con períodos de asistencia obligatoria posformación), verificar que en todos los Centros Docente Asistenciales se cumplan las condiciones para el entrenamiento de los futuros especialistas de tal manera que den efectivamente garantías a la población usuaria, y transparentar todos los sistemas de selección $n^{6}$. En cualquier caso, es necesario avanzar en este sentido en el corto plazo, por lo que la incorporación de médicos en especialización en ORL al sistema CONE (Concurso Nacional de Especialistas) es un paso positivo que permitirá a los médicos realizar sus estudios con un sueldo asegurado, y vincularlos al SP al egresar, aun cuando este sistema es susceptible de ser modificado a futuro, mejorando su flexibilidad.
Dentro de las principales ventajas de trabajar en el sector público se mencionan la experiencia quirúrgica que se adquiere, el aporte a la salud pública y docencia, y la seguridad previsional. Dentro de las desventajas se menciona la baja remuneración, el riesgo médico legal, y la alta exigencia administrativa. Estos factores deberían ser considerados a la hora de diseñar las políticas de vínculo y retención de los ORL en el sector público de salud, sobre todo considerando que la tendencia preponderante en la actualidad de los médicos es preferir el sistema privado ${ }^{5}$.

Si revisamos lo sucedido en términos de trabajo en servicio público en España, se aprecia que dentro de lo establecido para lograr una regulación más precisa de los empleados públicos se incorporaron conceptos en su legislación como fomentar la empleabilidad de los trabajadores y la formación profesional, fomentar la contratación indefinida, y orientar los contratos a la formación y el aprendizaje del profesional ${ }^{8}$. Se menciona en nuestro país que la permanencia de especialistas en el servicio público de salud va a depender en gran medida de las remuneraciones, la tecnología, insumos y personal de colaboración necesarios para el desarrollo de su especialidad ${ }^{9,10}$. Este tipo de medidas, aunque han sido aplicadas en otros contextos, podrían sumarse a medidas basadas en las razones aportadas en este artículo para estimular a los ORL recién egresados, 0 incluso a los que ya se desempeñan, a vincularse de manera parcial con el SP.

Dentro de las desventajas del presente artículo, se encuentra que se logró obtener respuesta de solamente $43 \mathrm{ORL}$ de un total de 97 contactados. Por esta razón, estos resultados no deberían ser tomados como totalmente representativos de los ORL del país. Sin embargo, aporta información relevante para los tomadores de decisión (policy-

Tabla 4. Porcentaje de otorrinolaringólogos que trabajan en sector público de salud según año de egreso de la especialidad $(n=43)$

\begin{tabular}{|l|c|c|c|c|c|c|c|c|c|c|c|}
\hline $\begin{array}{l}\text { Año de } \\
\text { egreso }\end{array}$ & 2003 & 2004 & 2005 & 2006 & 2007 & 2008 & 2009 & 2010 & 2011 & 2012 & valor de p \\
\hline $\begin{array}{l}\text { Trabaja } \\
\text { en } \\
\text { sector } \\
\text { público }\end{array}$ & $27,3 \%$ & $36,4 \%$ & $30 \%$ & $55,6 \%$ & $62,5 \%$ & $70 \%$ & $66,7 \%$ & $50 \%$ & $83,3 \%$ & $100 \%$ & 0,003 \\
\hline
\end{tabular}


makers) sobre el estado actual de la especialidad en el país. Llama especialmente la atención el tiempo relativamente constante de vínculo de gran parte de los ORL con el SP (3 años y 10 meses en promedio), lo que sumado al hecho que existe una asociación significativa entre año de egreso de la generación y proporción de trabajo en el sector público (con menos ORL trabajando en el SP a mayor tiempo de egreso de la especialidad), y a que la principal razón argumentada para ingresar a trabajar al SP y la primera ventaja de trabajar en este era el adquirir experiencia, sugiere que quizás muchos $\mathrm{ORL}$ recién egresados se quedan trabajando en el SP para terminar de formarse quirúrgicamente, y que luego de un tiempo al no tener mayores estímulos, renuncian para dedicarse a trabajar en el sistema privado. En cualquier caso, lo ideal sería analizar este fenómeno con un abordaje científico cualitativo.

En conclusión, es importante considerar estos factores al diseñar las políticas de vínculo y retención de ORL en el sector público de salud, y se debería fomentar este vínculo para ayudar a disminuir la brecha de acceso a especialistas ORL entre el sistema público y privado de salud.

\section{BIBLIOGRAFÍA}

1. Cardemil F, Barría t, Rahal M, Rodríguez R. Cantidad y distribución de otorrinolaringólogos en los sectores público y privado de salud en Chile. Revista Chilena de Otorrinolaringología 2013; 73(1): 25-32.

2. Rosselot E, Mateluna E, Estevez A, Radrigán
ME. Acreditación de título de profesionales e instituciones formadoras: el caso de Chile. Rev Méd Chile 1998; 126: 715-24.

3. Banco Mundial y Gobierno de Chile. Estudio de brechas de oferta y demanda de médicos especialistas en Chile. Informe. Diciembre 2009.

4. Román 0, Señoret M. Estado actual de las especialidades médicas en Chile; realidad en el sistema público no municipalizado. Rev Méd Chile 2008; 136: 99-106.

5. Román 0, Feliú M, Echavarría L. Formación de médicos especialistas en el Sistema de Servicios de Salud de Chile: Su evolución en el período 2007-2010. Rev Méd Chile 2011; 139 (6): 762-9.

6. Enríquez 0. Departamento de Formación y Acreditación Médica. Principios permanentes y actuales del departamento de formación y acreditación médica - Colegio Médico de Chile, 2011.

7. Colaboración Técnica Banco Mundial - Gobierno de Chile. Serie cuadernos de redes $N^{0} 31$. Estudio de Brechas de Oferta y Demanda de Médicos Especialistas en Chile. Subsecretaria de Redes Asistenciales. Departamento de Estudios de Recursos Humanos. Enero de 2010.

8. Cruz J. Hacia un nuevo modelo laboral en España. Derecho PUCP 2012; 68: 131-56.

9. Román 0, Acuña M, Señoret M. Disponibilidad de médicos en Chile al año 2004. Rev Méd Chile 2006; 134: 1057-64.

10. Salazar C, Cardemil F, Peña J. Estado Actual e Implicancias de la Acreditación de Escuelas de Medicina en Chile. Rev Méd Chile 2009; 137: 1126-7. 